Instituto Internacional de Investigación y Desarrollo Tecnológico Educativo INDTEC, C.A.

DOI: https://doi.org/10.29394/Scientific.issn.2542-2987.2019.4.E.7.116-135

OAl-PMH: http://www.indteca.com/ojs/index.php/Revista Scientific/oai

Artículo Original / Original Article

\title{
Comunicación interna en la calidad de la gestión administrativa Liceo Técnico Amelia Courbis, Talca 2018
}

Autoras: Amely Dolibeth Vivas Escalante Universidad Miguel de Cervantes, UMC amelydolly@hotmail.com Talca, Chile http://orcid.org/0000-0002-5791-8619

Paulina Alicia Saavedra Moraga Universidad Miguel de Cervantes, UMC saavedrapaulina@hotmail.com Talca, Chile http://orcid.org/0000-0003-2468-6679

\section{Resumen}

El presente estudio se enmarcó en una investigación no experimental. El universo constituido por ciento cincuenta (150) profesores y la muestra por setenta y cinco (75) profesores del nivel de la institución antes mencionada. El diseño de la investigación es descriptivo, transversal. Los datos fueron recolectados a partir de dos cuestionarios estructurados tomando en cuenta: la comunicación interna, en sus formas y tipos de comunicación descendente, ascendente, horizontal. La calidad de la gestión administrativa, orientada a planificar, organizar, integrar, direccionar y controlar, el cual se administró a los 75 profesores de la muestra. La hipótesis de investigación: La comunicación interna se relaciona con la calidad de la gestión administrativa en el Liceo Técnico Amelia Courbis. Los resultados inducen a rechazar la hipótesis nula y aceptar la hipótesis investigada, porque con el estadístico aplicado de prueba $\mathrm{R}$ de $\mathrm{Ch} 2$ el resultado de correlación se muestra con un índice de, 0.953, es decir 95.3\%, con un índice de libertad de 0,047 o 4.7\%, con lo que se valida la hipótesis alterna: La comunicación interna se relaciona con la calidad de la gestión administrativa en el Liceo Técnico Amelia Courbis, Talca.

Palabras clave: comunicación; organización; gestión.

Cómo citar este artículo:

Vivas, A., \& Saavedra, P. (2019). Comunicación interna en la calidad de la gestión administrativa Liceo Técnico Amelia Courbis, Talca 2018. Revista Scientific, 4(Ed. Esp.), 116-135, e-ISSN: 25422987. Recuperado de: https://doi.org/10.29394/Scientific.issn.2542-2987.2019.4.E.7.116-135

Fecha de Recepción: 08-05-2019
Fecha de Aceptación: 15-07-2019
Fecha de Publicación: 05-09-2019 


\title{
Internal communication in the quality of administrative management Amelia Courbis Technical High School, Talca 2018
}

\begin{abstract}
The present study was framed in a non-experimental investigation. The universe consists of one hundred and fifty (150) professors and the sample by seventy-five (75) professors of the level of the aforementioned institution. The research design is descriptive, transversal. The data were collected from two structured questionnaires taking into account: internal communication, in its forms and types of descending, ascending, horizontal communication. The quality of administrative management, aimed at planning, organizing, integrating, directing and controlling, which was administered to the 75 professors in the sample. The research hypothesis: Internal communication is related to the quality of administrative management at the Amelia Courbis Technical High School. The results induce to reject the null hypothesis and accept the investigated hypothesis, because with the applied test statistic $\mathrm{R}$ of Ch2 the correlation result is shown with an index of 0.953 , that is to say $95.3 \%$, with a freedom index of 0.047 or $4.7 \%$, which validates the alternative hypothesis: Internal communication is related to the quality of administrative management at the Amelia Courbis Technical High School, Talca.
\end{abstract}

Keywords: communication; organization; management.

\footnotetext{
How to cite this article:

Vivas, A., \& Saavedra, P. (2019). Internal communication in the quality of administrative management Amelia Courbis Technical High School, Talca 2018. Revista Scientific, 4(Ed. Esp.), 116135, e-ISSN: 2542-2987. Recovered from: https://doi.org/10.29394/Scientific.issn.25422987.2019.4.E.7.116-135
}

Date Received: 08-05-2019
Date Acceptance: 15-07-2019
Date Publication: 05-09-2019 


\section{Introducción}

Hoy en día transmitir información se ha convertido en un proceso de retroalimentación en las instituciones o empresas, para Katz y Khan (2014a), es: "la transmisión de mensajes, es decir hay una interacción constante dentro de las organizaciones, por ello, son un sistema abierto, donde el dinamismo social insta a transformaciones en el contexto donde se encuentra el hombre" (pág. 90).

Por otro lado, para Garrido (2007), citado por Mendoza y López (2015): "la estrategia es el centro neurálgico de la empresa, es el objeto de conocimiento del pensamiento estratégico" (pág. 87). Por ende, utilizar la comunicación activa, es esencial para ayudar a la coordinación y el control de las responsabilidades de los trabajadores que conforman dicha organización, a fin de crear un ambiente apropiado a nivel laboral y estratégico.

Hoy en día, en las instituciones educativas es esencial el intercambio entre quienes hacen vida dentro de dichas instalaciones, lo que incide directamente en el aprendizaje significativo de los educandos, la calidad de los procesos de enseñanza, proceso evaluativo entre otros aspectos de la vida laboral.

En ese sentido, en el Liceo Técnico Amelia Courbis, Talca, hay mucho trabajo por realizar con todas las instituciones educativas que lo conforman, pues este problema de comunicación interna entre los miembros que cohabitan en dichas instancias, sobre todo se observa en los eventos educativos tanto dentro como fuera del ente educativo donde participan los estudiantes, en las relaciones entre los docentes y también entre la plana jerárquica.

Dentro de ese proceso educativo la comunicación es bidireccional entre quien emite el mensaje y quien lo recibe, donde muchas veces los roles difieren de acuerdo al grado de compromiso, lamentablemente muchas veces este proceso se interrumpe porque se da una información, pero sin que haya 
comunicación. Para Luhmann (2013), el proceso comunicacional depende de: "reconocer la conexión entre el dominio de la información, el manejo de habilidades para comunicarse y finalmente comprender los mensajes a fin de establecer las relaciones interpersonales" (pág. 154).

Considerando la gestión, Medina (2019), resalta que:

La gestión se sintetiza en el desarrollo de cuatro funciones básicas para coordinar de forma eficaz y eficiente el trabajo de los demás, utilizando para ello las gestiones dirigidas a planear, organizar, dirigir, control, que son propios de la gestión estratégica en la organización. De este modo, el carácter general, flexible y de largo plazo, que se dan a nivel alto e institucional; para desplegar de manera táctica las acciones que se proyectan a mediano plazo en los niveles intermedios y operacionales, donde se reflejan los detalles para alcanzar los objetivos a corto plazo a nivel funcional (pág. 280).

Por consiguiente y de acuerdo a lo expresado por el mencionado autor, para que se desarrolle un proceso comunicativo interno la información debe ser expresada y esta expresión comunicativa ha de ser comprendida por todos. Así, en el Liceo Técnico Amelia Courbis, Talca, hay con suma urgencia la necesidad de una comunicación interna adecuadamente en las organizaciones educativas, tanto para la institución educativa y el personal que laboran en ellas, por ello debemos considerar:

Los procesos comunicativos de acuerdo a Katz y Kahn (2014b), que se llevan a cabo en la institución educativa: "buscan el logro de los objetivos organizacionales mediante mensajes que fluirán eficientemente, produciendo relaciones apropiadas, ambiente laboral agradable" (pág. 54); es así como uno de estos subsistemas es incentivados para el cumplimiento de sus tareas logrando los resultados esperados por el sistema, en este caso particular la institución educativa, Liceo Técnico Amelia Courbis.

Por otra parte, este proceso comunicativo dentro de las organizaciones induce a las transformaciones conducidas por el razonamiento 0 
discernimiento de manera conlleve a adaptarse a ese nuevo contexto, de allí que en el Liceo Técnico Amelia Courbis, permitirá mantener la información, integración y la motivación para el logro de los objetivos organizacionales a partir del trabajo. De este modo, la comunicación interna en la institución educativa va a inducir a los autores de la organización en su visión y misión, a ser creativos, motivados e integrados al desarrollo personal.

De esta manera, la comunicación interna conllevará a mejorar la calidad laboral, calidad educativa, manejando las herramientas, técnicas y procedimientos que incrementen la productividad y competitividad del entorno, es decir, la calidad del producto ofrecido y la calidad del trabajo ejercido por su capital humano. El nexo que interrelaciona la comunicación interna en el Liceo Técnico Amelia Courbis es necesaria, en el ámbito de la calidad total, el manejo de un sistema de información a todos los niveles, donde el elemento fundamental, es decir, el empleado, quien debe internalizar los aspectos de la calidad, a fin de implantarla, asumirla y desarrollarla, razón por la cual, es fundamental a nivel educativo, la comunicación interna, para los estudiantes, docentes, personal de servicio, administrativos.

En ese contexto el rol de la comunicación interna a nivel de la calidad de la gestión administrativa en el Liceo Técnico Amelia Courbis, ubicado en Talca, Chile, no se escapan de la situación antes planteada, exaltando todos los aspectos que deben tomarse en cuenta en el manejo administrativo, así como los problemas propios que enfrenta la comunidad educativa. Permita este trabajo de investigación tomar conciencia de problema que se está observando y afrontarlo, a partir de acciones que conlleven a acrecentar aciertos y corregir errores de la gestión administrativa. El papel de las redes educativas es muy valioso y se debe dar cumplimiento a lo establecido en el Reglamento de organización y funciones de las redes educativas, publicadas por el Ministerio de Educación en el año 2009. 


\section{Desarrollo}

\subsection{La comunicación interna}

A nivel empresarial la comunicación interna es una herramienta proyectada al interior de la organización, a fin de producir un ambiente armonioso y productivo, para alcanzar un fin, al interpretar los mensajes. Esta comunicación permitirá relacionarse las personas entre los diferentes niveles de mando.

Por otra parte, la comunicación interna motiva al capital humano a tener pertenencia promoviendo el trabajo en equipo a fin de lograr los objetivos de la empresa. Además, con este tipo de comunicación se creará una atmósfera muy positiva en cuanto al manejo de la información, los propósitos y relevancia de cada acción ejecutada por los empleados, posibilitando la toma de decisiones individuales y grupales.

De allí que la comunicación o la transmisión de información será según Capriotti (1998): "un claro matiz dirigista, en el cual la comunicación, o mejor dicho, la transmisión de la información es sólo descendente" (pág. 1).

Actualmente, este tipo de comunicación valora el medio que emite la información dentro de la organización, lo cual va a estar por encima de las relaciones interpersonales, es decir, se manejará informes internos de la empresa, entre otras modalidades que permitan informar sobre los objetivos a seguir por parte de la organización.

Además, la comunicación interna, busca la activación en cuanto a la participación, busca que los empleados colaboren, sugieran, comenten, es decir, los involucra en el logro de los objetivos planteados. De allí que los mensajes se dan de manera bidireccional en las formas: ascendente, descendente y horizontal, siempre en la búsqueda del diálogo, que permite la relación entre las personas, logrando el carácter comunicacional.

La importancia de la comunicación interna entre los empleados de una institución, donde ellos participen haciendo sugerencias o proponiendo 
diversas propuestas comunicativas, aun cuando estas instituciones no estén preparadas para aceptar este tipo de relaciones.

\subsection{Dimensiones de la comunicación interna}

\subsubsection{Dimensión Esencial}

El diálogo es el punto de inicio de los cambios organizacionales para dar respuestas a las múltiples metas propuestas. De acuerdo a Rivera, Rojas, Ramírez y Álvarez de Fernández (2005):

La comunicación es una herramienta de gestión, ya que contribuye al consenso entre el personal en las empresas, es decir, las organizaciones que mantienen sistemas de comunicación abiertos, da oportunidad al personal para que tome parte de los procesos que están orientados a la creación de los valores de la organización, alimentando así el sentido de pertenencia" (pág. 37).

Para una organización la esencia parte de la palabra, es la base teórica, le facilita la sustentación a cada espacio dentro de la organización, a fin de mantener su vivencia y supervivencia, porque las acciones inducen a metas, si se dosifica la palabra, se estructura la organización.

\subsubsection{Dimensión Operativa}

Dentro de la empresa, la comunicación interna debe trasmitir que todos los empleados manejen que deben hacer su trabajo de la manera correcta, está fase implica lo básico, operativo y esencial.

\subsubsection{Dimensión Estratégica}

En relación a la dimensión estratégica, el empleado debe conocer por qué realizan una u otra actividad en función de las metas propuestas por la organización, de esta manera será eficiente y efectivo su labor, todos los empleados sabrán hacia donde esta direccionado su trabajo. La comunicación 
estratégica debe encauzar a un solo fin y de esta forma, se logrará las metas apropiadas, la misión, visión y propósitos organizacionales.

El compromiso que adquiere el empleado por los objetivos tanto personales como de la empresa, implica reconocer la direccionalidad que requiere la organización, en consecuencia, siente que es tomado en cuenta, allí hay un valor agregado, que permite la visión prospectiva de su labor. El empleado siente que es motivado, su labor tiene una meta que se consigue con el trabajo en equipo, exaltando cual es la proyección de su tarea en función de los objetivos de la empresa.

A esto se adiciona, que la comunicación conduce a mediar los conflictos y malestares que puedan producirse, si al empleado no se le comunica estratégicamente, siente que sus tareas no tienen importancia sólo es por cumplir los designios de una persona en particular, más no es, para lograr objetivos institucionales.

\subsubsection{Dimensión Valorativa}

Cuando se hace referencia a la dimensión valorativa, indica que dentro de la organización se conduce al empleado para que realice sus actividades y tareas en sintonía con los objetivos y valores empresariales, de esta manera, el trabajador se siente tomado en cuenta porque lo que realiza es fundamental para la empresa.

Todo empleado dentro de la organización puede iniciar su proceso laboral con muchos deseos y ganas de hacerlo bien siempre, pero puede desvirtuarse esa motivación en la medida en que observa situaciones no claras dentro de la empresa, puede ser, de qué manera se adquieren los recursos económicos para la compra de la materia prima, la directora de la empresa es una persona corrupta, seguirá el empleado laborando dentro de esa empresa.

Dentro del campo organizacional debe ser comunicado los valores a fin de posteriormente demostrarse en la realidad, éstos no dependen del 
momento histórico en el cual se encuentra la empresa, son constantes nacieron con la organización, lo que puede variar son los objetivos, las tareas y los roles de los empleados porque estos esta supeditados a las necesidades. Pero toda empresa desde que se engendra parte con principios y los mismos deben ser tan sólidos que permanezcan en el tiempo, razón por la cual la comunicación debe permitir integrar, fortalecer y atraer a sus empleados a los objetivos empresariales.

\subsubsection{Dimensión Motivacional}

En toda organización la persona conoce sus funciones, como desarrollarlas, cuando llevarlas a cabo, pero entra en juego si desea realizarlas. En este momento la motivación, se convierte en el elemento fundamental, las comunicaciones se hacen efectivas entre los empleados generando en ellos: Orgullo por ser pieza clave de la organización, visión prospectiva de ascensos, considera que la empresa ofrece lo que cada empleado se merece, siente que es valorado, por ende, escuchado, lo consideran un ser humano con sus fortalezas y debilidades.

\subsubsection{Dimensión Aprendizaje}

A través de la comunicación se abre la oportunidad del feedback, entre el empleado y la organización, estableciendo como clave las mejoras en su trabajo. Dentro de la organización se debe abrir espacios de comunicación o diálogo a fin de ir mejorando de manera simultánea con la tarea que está realizando, por ello es necesario: Constantes momentos de diálogo fluido, debe la organización establecer que espera de cada empleado, darle la importancia que se merece cada empleado en función de su desempeño, la evaluación debe permitir acrecentar ciertos y corregir errores, nunca debe ser para cercenar o amedrentar al empleado.

El proceso de feedback debe ser direccionado por personas 
capacitadas para tal fin. La manera de retroalimentar los procesos debe ser visto como parte de la cultura organizacional de la empresa. La retroalimentación debe ser desarrollada por la persona indicada constantemente antes, durante y después de realizar las evaluaciones.

\subsubsection{Dimensión Inteligencia}

Según, Villasante (2017), la inteligencia se basa:

En la búsqueda constante de información y el análisis, proporciona los conocimientos que necesiten quienes establezcan la política y la estrategia, hacen planes y toman decisiones, y ejecutan las acciones. Se trata de ir descubriendo, con la mayor anticipación posible, las características y valoraciones de las diversas variables y acontecimientos que pueden afectar de forma significativa a las propias decisiones estratégicas, así como cuales son los riesgos internos o externos cuya materialización implique un trastorno grave en el funcionamiento de la actividad (pág. 1).

De esta manera, las personas participaran en el desarrollo del plan de acción, donde cada uno expondrá a partir de sus vivencias como lograr que cada estrategia se direccione hacia las metas.

\subsection{Funciones de la comunicación interna}

La comunicación interna tiene cuatro funciones esenciales para cualquier empresa, a saber: control, motivación, expresión emocional e información:

Control: dentro de una empresa el comportamiento de los empleados es controlado por los niveles jerárquicos, esto se evidencia cuando se le solicita al empleado que exponga cualquier situación que se presente, siguiendo los protocolos del caso, esa comunicación es un control y así sucesivamente, en el campo educativo cuando se solicita la planificación educativa a cada profesor, es un control para hacer un seguimiento a su labor 
académica.

Motivación: la motivación, es direccionada por la comunicación y en ella se aborda al empleado expresando lo bien que ejecuta su labor, resaltando donde están sus debilidades, fortalezas y oportunidades de manera que su desempeño sea satisfactorio y vaya concatenado con el logro de las metas de la organización. En el campo educativo la motivación se genera cuando el directivo acompaña el quehacer educativo y de esta manera estimula al docente sugiriendo donde puede mejorar sus técnicas, herramientas y procesos a fin de lograr aprendizajes significativos vivenciales con los estudiantes, por otra parte, a nivel administrativo, cuando se le expresa a una secretaria que bien realizó su trabajo pero que puede mejorar siguiendo estas pautas.

Expresión emocional: a partir de la comunicación las personas puede inferir sus estados emocionales, partiendo de los mismos se establecen las interacciones. A nivel educativo, se evidencia, por ejemplo, con el docente si se siente frustrado, insatisfecho por sus pagos laborales su proceso de enseñanza estará supeditado a aplicar estrategias de acuerdo a las necesidades e intereses de los estudiantes, lo cual desvirtúa el real trabajo pedagógico.

Información: necesaria para que los empleados tomen decisiones ante situaciones particulares, de esta manera se consideran los aspectos relevantes para dar respuesta a dichas situaciones.

Cada una de estas funciones son esenciales, ninguna es más preponderante que la otra, para que el trabajo se ejecute con eficacia debe controlarse las acciones de los miembros, consustanciado con la estimulación y motivación constante en su desempeño lo cual esta concatenado con lo emocional que conducirá a decisiones oportunas. 


\subsection{Importancia de la comunicación interna organizacional}

La participación de los empleados dentro de cualquier empresa es fundamental que sea tomada en cuenta por parte de los niveles jerárquicos para gestionar los problemas adecuadamente. Uno de los problemas que presenta la comunicación es la manera de interrelacionarse, porque no se manejan los canales y los mensajes no son transmitidos apropiadamente. En efecto, la conciencia de realizar comunicaciones internas fluidas es trascendentales hasta el punto que algunas empresas han propiciado espacios, plazos y procedimientos para que se ejecuten constantemente.

El sistema de comunicación interna será fluido en la medida que: La información necesaria de la empresa se le otorgue al personal. Debe establecerse la retroalimentación consensuada. Evitar los rumores a partir de lo emitido por la empresa como ente oficial. Evitar la duplicidad de trabajo a través de una comunicación efectiva. Además, se debe considerar los actores en la comunicación interna, que se dividen en dos grupos, a seguir:

Los líderes: la comunicación interna sensibilizará al personal, desarrollando el trabajo en equipo y estableciendo la confianza en cada uno de los niveles jerárquicos. A nivel educativo, parte del director o personal directivo quien debe ejercer el liderazgo y crear ese clima organizacional fundamental para el logro de metas.

Los empleados: a través de la comunicación interna, su trabajo será reconocido porque la empresa direccionará como debe hacerlo, tomando las decisiones oportunas. En el campo educativo, estará conformado por los docentes y demás personal que hace vida en las instituciones educativas.

La División de la comunicación interna, según su contenido, se divide en: Operacional: Interrelacionan la información referida al trabajo y Motivacional: Inducido al nivel de pertenencia y clima organizacional. 


\subsection{Comunicación: un ángulo socioconstructivista}

El plantear el problema sobre comunicación interna en el Liceo Amelia Courbis, permite esbozar diversas interpretaciones sobre comunicación además teorizar sobre el funcionamiento de la comunicación interna en las instituciones educativas. La perspectiva interpretativa insta sobre la comunicación interna basada en la teoría científica, es decir el socioconstructivismo, lo que conduce a opinar sobre un contexto real de la comunicación basada en la participación de dos personas comprendiendo lo expresado.

Considerando al autor Watzlawick (2014): "la comunicación va a depender de la interacción entre individuos, es decir, se presenta una visión relacional y de contenido" (pág. 65). El hombre se comunica en función de los ámbitos donde se desenvuelve el ser humano, es decir, la comunicación no sólo tendrá implícito la relación, el contenido sino el contexto donde se desarrolla la misma.

\subsection{La responsabilidad del mando intermedio}

De acuerdo a los niveles intermedios de mando el Director(a) es el garante de la comunicación interna y su apoyo son los subdirectores ya que se manejan en una segunda instancia, pero en la praxis real, la comunicación interna la inducen los coordinadores de área porque:

Estos realizan reuniones de área a de discrepar sobre contenidos programáticos y otros, luego realizan otros encuentros ya sean semanales, quincenales o mensuales con los directivos de cada nivel a fin de solventar situaciones que se presenten de manera mancomunada, luego el director bajará dicha información al personal docente. El coordinador motivará al personal tomando en cuenta los aspectos comunicativos en función de:

La información: el feedback, tiene como propósito orientar para el logro de objetivos preestablecidos, razón por la cual se debe puntualizar al 
empleado lo que debe hacer, cómo y por qué, de esta manera sentirá seguridad por lo que va a realizar.

La agenda diaria: esta implica jerarquizar las tareas de acuerdo al grado de relevancia, indicando mediante mensajes precisos lo que se desea lograr.

Asignación de responsabilidades: cada empleado tiene habilidades y destrezas para desarrollar un trabajo específico, razón por la cual debe ser orientado en los diferentes encuentros su responsabilidad.

Evaluación de desempeño: permite corregir errores y acrecentar aciertos, de esta manera debe aplicarse el feedback y el acompañamiento de aula en el caso de los docentes. En este sentido, se puede decir que la estrategia en el proceso educativo debe ser siempre motivadora a fin de lograr las metas propuestas por la institución.

Delegar tareas: se produce cuando la actividad laboral la ejecuta un compañero. La tarea de motivar al ser delega es muy efectiva, porque delimita la actividad a ejecutar, especificando cómo hacerlo a su vez, se plantea al colaborador que se espera de él. En este proceso de intercambio de opiniones s evidencia la comprensión de la que se va a ejecutar y los recursos con que cuenta para la misma.

Asertividad: se entiende por el respeto a los pensamientos y creencias de otras personas, de allí que esta habilidad los empleados permite el respeto y la igualdad entre los seres humanos, porque, aunque la persona no esté de acuerdo con el planteamiento de su compañero, debe respetarlo. De esta manera, se transmite información entre los empleados y se motiva constantemente.

\section{Metodología}

En cuanto a lo metodológico, se desarrolló a nivel explicativo, un estudio de causa y efecto en el cual se indaga sobre la Comunicación interna en la 
calidad de la gestión administrativa Liceo Técnico Amelia Courbis, Talca 2018.

En la recolección de datos se utilizó como técnica: la encuesta y como instrumento el cuestionario, contando con una población de setenta y cinco (75) docentes del año escolar 2018.

\section{Resultados}

Luego de aplicados los instrumentos a los docentes, se obtiene que la comunicación interna posee las frecuencias que se muestran en la tabla 1 y gráfico 1. En cada una de ellas se observa que la comunicación interna es considerada por la muestra seleccionada como ineficaz por la mayoría de los docentes, estos resultados le dan relevancia a la investigación.

Tabla 1. Frecuencia de respuestas.

\begin{tabular}{|l|c|c|}
\hline COMUNICACIÓN INTERNA & FRECUENCIA & PORCENTAJE (\%) \\
\hline Eficaz & 41 & 41.0 \\
\hline Ineficaz & 59 & 59.0 \\
\hline TOTAL & $\mathbf{1 0 0}$ & $\mathbf{1 0 0 . 0}$ \\
\hline
\end{tabular}

Fuente: Las Autoras (2019).

Gráfico 1. Respuesta de los docentes encuestados.

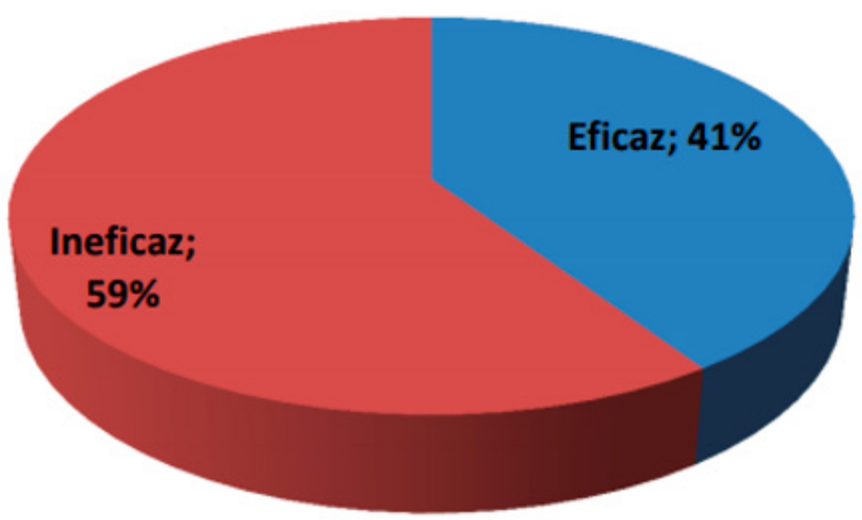

Fuente: Las Autoras (2019).

En la tabla 1 y gráfico 1 , se puede evidenciar que la mayoría de los 
docentes indican que la comunicación interna no se aplica en la institución y no cumple con lo indicado en la bibliografía consultada que una buena gestión administrativa posee una buena comunicación interna ya que permite facilitar los procesos, mejorando la calidad en la gestión mediante.

De acuerdo con la hipótesis general aplicando la prueba $\mathrm{R}$ de $\mathrm{Ch} 2$ el resultado de correlación se muestra con un índice de, 0.953, es decir 95.3\%, con un índice de libertad de 0,047 o 4.7\%. Ella llega a la siguiente conclusión en su investigación: Enfatizar sobre la comunicación interna en las instituciones educativas, debido a la relación que tiene la cultura, el clima organizacional y el nivel de compromiso con los objetivos y metas de la institución.

Se menciona que la comunicación descendente se relaciona con la calidad de la gestión administrativa en el Liceo Técnico Amelia Courbis. Así, la aplicación de la prueba $\mathrm{R}$ de $\mathrm{Ch} 2$ el resultado de Correlación se muestra con un índice de 0,975, es decir 97.5\%, con un índice de libertad de ,025 o $2.5 \%$, la comunicación ascendente se relaciona con la calidad de la gestión administrativa. Describe como la comunicación se relaciona con el trabajo en equipo para la toma de decisiones, donde se realce la productividad, además se den alianzas estratégicas para evitar las barreras organizacionales, finalmente proponer el trabajo en equipo.

En ese sentido se puede afirmar que la comunicación ascendente debería presentarse en los diversos niveles de manera participativa y democrática.

La comunicación ascendente donde se manejan las conciliaciones, mediaciones, apelaciones, asesorías, entre otras. Los resultados nos describen que al aplicar la prueba R de Ch2 el resultado de correlación se muestra con un índice de 0,976 , es decir $97.6 \%$, con un índice de libertad de 0,024 o $2.4 \%$, se ha establecido que la comunicación horizontal se relaciona con la calidad de la gestión administrativa. 


\section{Conclusión}

Es importante resaltar la opinión que concuerda con los resultados obtenidos, que una buena comunicación entre todos los empleados de la empresa, dejando de lado el perjuicio de darle mayor importancia, entre las personas de mayor jerarquía en la institución donde se trabaja, lo que no ayuda el desarrollo de la institución. Lo que se obtiene al final es el éxito institucional y la calidad. Por otro lado, si tomamos en cuenta la opinión de Martinet (2012), quién sostiene: "la comunicación son los signos para la transmisión del mensaje en los seres humanos relacionados entre sí" (pág. 163). Al analizar los resultados se ha establecido una relación entre comunicación horizontal y calidad de la gestión administrativa. Debemos tomar en cuenta que las informaciones que se desprenden de la red de comunicación tienen un significado preponderante en las interacciones humanas.

\section{Referencias}

Capriotti, P. (1998). La comunicación interna. Argentina: Reporte C\&D Capacitación y Desarrollo, (13). Recuperado de:

http://www.bidireccional.net/Blog/Comunicacion Interna.pdf

Katz, D., \& Kahn, R. (2014a,b). Psicología Social de la Organizaciones. México: Editorial Trillas.

Luhmann, D. (2013). Comunicación Empresarial: Plan Estratégico como herramienta gerencial. Centro de investigaciones de la Comunicación Corporativa Organizacional. Bogotá, Colombia: ECOE Ediciones; Universidad de la Sabana.

Martinet, A. (2012). Economía de los cambios fonéticos. Madrid, España: Editorial Gredos.

Medina, J. (2019). Gerencia Tecnológica de la Información y Comunicación en las Organizaciones Sociales. Revista Scientific, 4(12), 275-289, e-ISSN: 2542-2987. Recuperado de: 
https://doi.org/10.29394/Scientific.issn.2542-2987.2019.4.12.14.275-

$\underline{289}$

Mendoza, D., \& López, D. (2015). Pensamiento estratégico: centro neurálgico de la planificación estratégica que transforma la visión en acción. Económicas CUC, 36(1), 81-94, e-ISSN: 2382-3860. Recuperado de:

https://dialnet.unirioja.es/servlet/articulo?codigo $=5156212$

Rivera, A., Rojas, L., Ramírez, F., \& Álvarez de Fernández, T. (2005). La Comunicación Como Herramienta de Gestión Organizacional. Negotium: revista de ciencias gerenciales, 1(2), 32-48, e-ISSN: 18561810. Recuperado de:

https://dialnet.unirioja.es/servlet/articulo?codigo $=2256686$

Villasante, J. (2017). Inteligencia, Comunicaciones y Seguridad. cronicaseguridad.com. Granada, España: Centro Andaluz de Estudios y Entrenamiento, S.L. Recuperado de:

https://cronicaseguridad.com/2017/09/04/inteligencia-comunicacionesseguridad/

Watzlawick, P. (2014). Comunicación Interna Gestión de Empresas. España: Ediciones Vértice, S.A. 


\section{Amely Dolibeth Vivas Escalante}

e-mail: amelydolly@hotmail.com

Nacida en San Cristóbal, Venezuela, el 3 de enero del

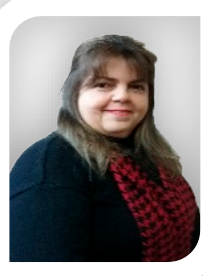
año 1970. Docente Académico-Investigador con PostDoctorado en Estudios Libres; Doctora en Ciencias de la Educación, titulada en la Universidad Fermín Toro (UFT); Magister en Planificación Educativa de la Universidad Pedagógica Experimental Libertador (UPEL). Especialista en Evaluación Educacional en la Universidad Pedagógica Experimental Libertador (UPEL); Licenciada en Educación Mención Matemáticas, en la Universidad de Los Andes (ULA); Ponente en diversas Conferencias a nivel de Educación. Docente a nivel doctoral. 


\section{Paulina Alicia Saavedra Moraga}

e-mail: saavedrapaulina@hotmail.com 